\title{
Uso de modelo experimental de anastomose látero-lateral microcirúrgica no ensino de habilidades cirúrgicas na graduação
}

\author{
Use of experimental model of latero-lateral end microsurgical anastomosis on teaching of \\ surgical skills in graduation
}

Matheus Silva Garbin ${ }^{1}$, Aline Riquena da Silva ${ }^{1}$, Sarah do Valle Studart ${ }^{1}$, Pedro Luiz Squilacci Leme ${ }^{2}$

\section{Resumo}

Introdução: A anastomose arteriovenosa latero-lateral é um procedimento complexo para acadêmicos de medicina, pois requer treinamento repetitivo. Objetivo: Comparar $o$ aprendizado de alunos do quinto e décimo semestres da graduação de medicina, após treinamento de anastomose arteriovenosa latero-lateral, em modelo para treinamento que utiliza línguas bovinas. Materiais e Métodos: Estudo realizado na Universidade Nove de Julho, São Paulo, SP, Brasil, com a participação de 20 graduandos de medicina (dois grupos de dez alunos, de quinto e décimo semestres). No primeiro dia os alunos assistiram aula sobre a técnica da anastomose arteriovenosa latero-lateral e realizaram o procedimento, sendo avaliados dez critérios quanto à realização do procedimento. Os grupos treinaram durante quatro sessões de duas horas e foram novamente submetidos à mesma avaliação. As notas médias foram analisadas pelo teste T de Student. Resultados: O grupo de quinto semestre obteve média de 3 pontos na avaliação 1 e 5 pontos na avaliação 2 ( $p=0,08393$ ). O grupo de décimo semestre obteve média de 5 pontos na avaliação 1 e 7 pontos na avaliação 2 ( $p=0,08393)$. Conclusões: Comparando o desempenho dos alunos de quinto e décimo semestre, após treinamento específico da anastomose vascular proposta, houve melhora na nota dos dois grupos, mas não houve significância estatística quando os valores foram comparados.

1. Acadêmicos do Curso de Medicina da Universidade Nove de Julho

2. Professor do Curso de Medicina da Universidade Nove de Julho. Professor Assistente da Faculdade de Ciências Médicas da Santa Casa de São Paulo

Trabalho realizado: Universidade Nove de Julho. Faculdade de Medicina

Endereço para correspondência: Pedro Luiz Squilacci Leme. Av. das Nações Unidas, 561, ap. 52. 09726-110 - São Bernardo do Campo - SP - Brasil. Tel. / Fax: (11) 4330-1977 - e-mail: gorableme@uol.com.br
Descritores: Anastomose arteriovenosa, Microcirurgia, Educação Médica, Simulação, Ensino, Bovinos, Cirurgia geral.

\begin{abstract}
Introduction: Latero-lateral end arteriovenous anastomosis is a complex procedure for medical students because it requires repetitive training. Objective: To compare the learning of students of the fifth and tenth semesters of medical graduation after training lateral-lateral arteriovenous anastomosis using bovine tongues. Materials and Methods: study developed at the Universidade of Nove de Julho, São Paulo, SP, Brasil, with the participation of 20 medical students (two groups of ten students, fifth and tenth semesters). On the first day the students attended a class about lateral arteriovenous anastomosis and performed the procedure, under evaluation of the technique based on ten criteria. The groups trained during four sessions of two hours and were again submitted to the same evaluation criteria. The mean scores were analyzed using Student's $T$ test. Results: The group of the fifth semester obtained an average of 3 points in the evaluation 1 and 5 points in the evaluation 2 ( $p=0,08393)$. The tenth semester group obtained an average of 5 points in the 1 assessment and 7 points in the evaluation $2(p=0.08393)$. Conclusions: Comparing the performance of the fifth and tenth semester students, after specific training of the proposed vascular anastomosis, there was improvement in the score of the two groups, but there was no statistical significance when the values were compared.
\end{abstract}

Keywords: Arteriovenous anastomosis, Microsurgery, Medical Education, Simulation, Teaching, Cattle, General surgery.

\section{Introdução}

O treinamento e simulação para o aprendizado técnico em cirurgia são essenciais no ensino, pois, a partir 
da explicação e demonstração, seguidos de prática repetitiva, permitem a aquisição da habilidade motora, seguindo a teoria de Fitts-Posner - cognição, integração e automatização da habilidade adquirida ${ }^{(1)}$.Para as simulações cirúrgicas, existem diferentes métodos que permitem treinar desde pequenos procedimentos, ou etapas de uma cirurgia, até cirurgias completas, utilizando materiais e aparelhos de diferentes custos ${ }^{(2-3)}$.

A iniciativa atual é diversificar o uso dos modelos de ensino já existentes para continuar a tendência na redução do uso de animais, bem como fortalecer a cultura do uso racional de animais em estudos experimentais ${ }^{(4)}$. Dentre os modelos de ensino mais usados hoje, os chamados "modelos de bancada", são de interesse por utilizarem materiais inanimados - artificiais (estruturas de borracha ou espuma) ou biológicos (línguas bovinas e outras vísceras animais) - de baixo custo $^{(5)}$, mas que permitem o ensino de princípios básicos de cirurgia, adequando-se às necessidades dos graduandos em medicina ${ }^{(6)}$.

Um modelo de bancada de uso frequente na graduação é o treino de suturas em língua bovina ${ }^{(7)}$. A fim de ampliar os usos do modelo, a língua bovina pode ser empregada testando o ensino de diferentes técnicas, por exemplo, a anastomose arteriovenosa latero-lateral - cuja confecção caracteriza-se pela complexidade elevada para acadêmicos de medicina, pois requer treinamento repetitivo.

O ensino de anastomoses vasculares é um tema já validado em programas de residência em cirurgia vascular, pois esta técnica é uma das primordiais para o dia-a-dia da especialidade ${ }^{(8-9)}$. Entretanto, a anastomose arteriovenosa latero-lateral é pouco ensinada em graduação, portanto, avaliar a curva de aprendizado desta técnica em graduandos em medicina, configura uma inovação do uso do modelo de bancada com línguas bovinas.

\section{Objetivo}

Comparar a curva de aprendizado de alunos do quinto e décimo semestres da graduação de medicina após treinamento de anastomose arteriovenosa latero-lateral, em modelo de ensino utilizando línguas bovinas.

\section{Materiais e Métodos}

Modelo executado no laboratório de habilidades cirúrgicas avançadas no Núcleo Integrado de Simulação Doutor Luiz Roberto Barradas Barata (NIS), da Universidade Nove de Julho, São Paulo, SP, Brasil, com a participação de 20 graduandos de medicina da Universidade Nove de Julho (grupo de 10 alunos de quinto semestre e grupo 10 alunos de décimo semes- tre). Foram usadas línguas bovinas para o ensino e treinamento da técnica.

Os alunos foram selecionados pois ao $5^{\circ}$ semestre, o graduando já passou pelo curso de técnica operatória, assim como o graduando de $10^{\circ}$ já cursou o estágio cirúrgico no internato. Nenhum dos grupos passou por treinamento prévio na técnica proposta pelo estudo.

No primeiro dia do estudo, todos os alunos assistiram a uma aula orientadora, ministrada por um professor de técnica operatória, sobre a técnica da anastomose arteriovenosa latero-lateral e realizaram o procedimento pela primeira vez, sendo avaliados com base em dez critérios elaborados para este estudo (avaliação 1). Os dois grupos treinaram por um mês, sob supervisão do professor que os orientou na aula inicial, numa frequência de uma vez por semana, durante duas horas em cada sessão, totalizando oito horas.

O procedimento realizado consistia em: dissecar a artéria e veia linguais, cateterizar ambos os vasos com cateter agulhado $25 \mathrm{G}$ (diâmetro $=0,51 \mathrm{~mm}$ ) e infundir solução salina. Criar incisões de $0,5 \mathrm{~cm}$ nas faces laterais de ambos os vasos, aproximá-los e suturá-los utilizando fio de sutura de polipropileno 6-0, com tensão e disposição adequada. Após as suturas, a solução era infundida para avaliação de extravasamentos. Ao longo de todo procedimento, a apreensão dos vasos deveria ser feita pelas adventícias, sem lesar as camadas média e íntima.

Terminando o treinamento de oito horas, os grupos realizaram o procedimento e foram submetidos à avaliação 2 seguindo os mesmos critérios e as notas médias de cada grupo foram analisadas pelo método T de Student.

\section{Resultados}

O grupo de quinto semestre obteve média de 3 pontos na avaliação 1 e média de 5 pontos na avaliação 2 ( $p=0,08393)$. O grupo de décimo semestre obteve média de 5 pontos na avaliação 1 e 7 pontos na avaliação 2 ( $\mathrm{p}=0,08393)$.

\section{Discussão}

O ensino de cirurgia baseado em treinos e simulações em laboratório é a melhor forma de fornecer ao aluno um modo seguro para desenvolver suas habilidades deliberadamente sem consequências a nenhum paciente. As metodologias utilizadas se transformaram e adaptaram desde a implementação dos treinamentos instituída por Halsted há cerca de um século(10).

O método primordial de treinamento envolve o uso de cadáveres e de animais vivos. Os dois modelos têm vantagem pelo realismo conferindo ganho de habilidade ao aluno que os utilizar para seus estudos 


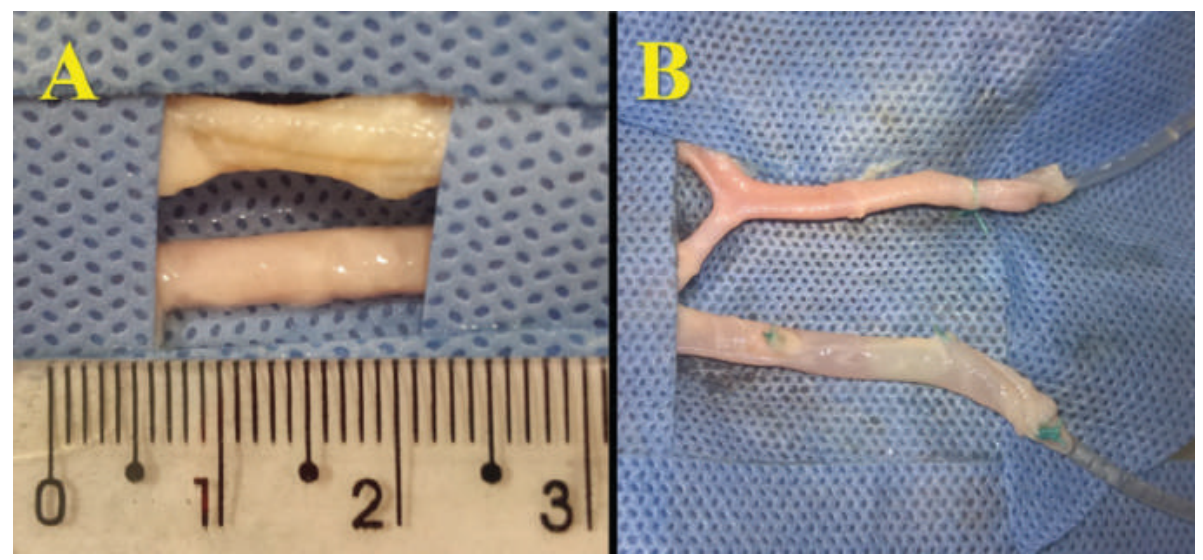

Figura 1 - A. dissecção da artéria e veia lingua; B. cateterização dos vasos e infusão de solução salina.

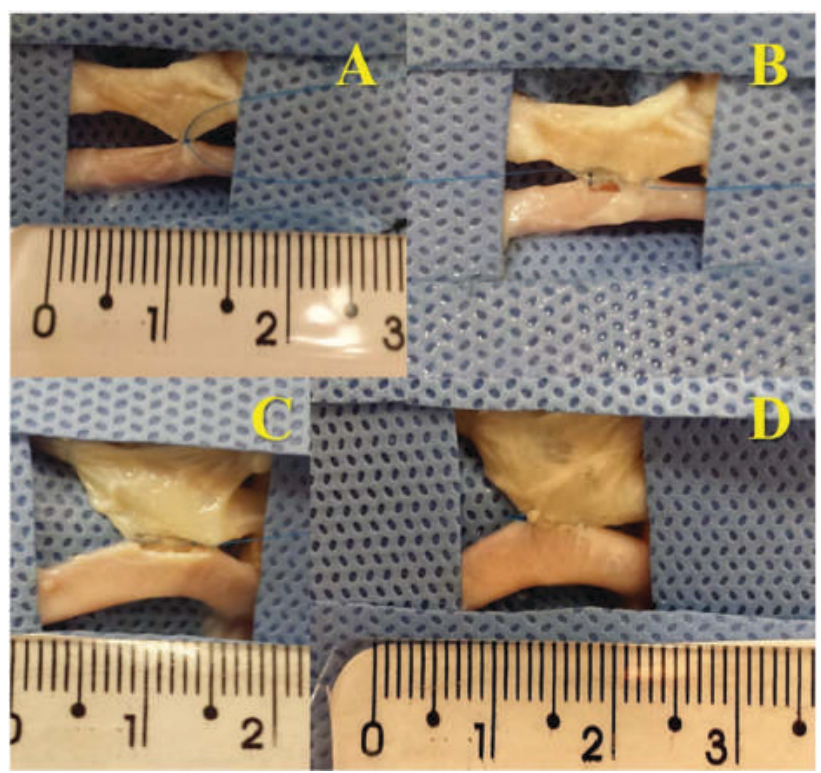

Figura 2 - A, B, C e D. sequência de suturas e realização da anastomose após treinamento. Anastomose realizada após treinamento.

e treinos, contudo, são demasiado custosos além de carregarem implicações éticas e legais que dificultam seu uso em laboratórios de treino ${ }^{(11)}$.

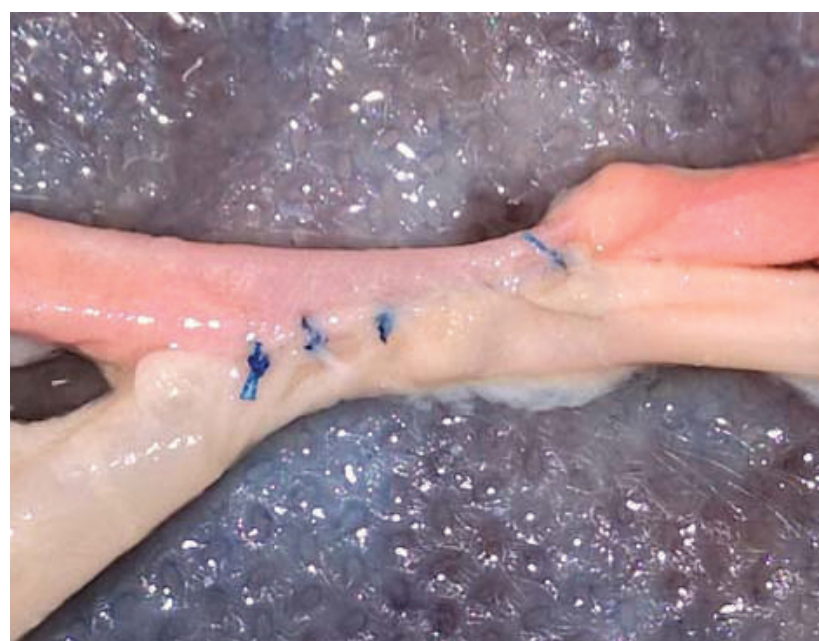

Figura 3 - Anastomose realizada após a aula inicial, antes do treinamento.

Sobre as implicações no uso de animais em experimentação, a bioética regulamenta o uso destes no Brasil por meio da lei Arouca (Lei no $11.794 / 08)^{(12)}$ que em seus artigos confere proteção ao uso dos animais em experimentação, e além disso, impõe os princípios dos "três R's" (replace, reduce, refine), que dizem respeito à substituição e redução do uso animal em pesquisa,

\section{Critérios de avaliação.}

\begin{tabular}{lcc}
\multicolumn{1}{c}{ Critérios de avaliação } & Sim & Não \\
1. Amparou os vasos com a pinça pela adventícia & 1 & 0 \\
2. Dissecou os vasos sem lesionar circulação colateral & 1 & 0 \\
3. Seccionou a parede do vaso sem transfixá-lo & 1 & 0 \\
4. Realizou secções compatíveis em tamanho & 1 & 0 \\
5. Realizou secções compatíveis em localização & 1 & 0 \\
6. Suturou as paredes vasculares de forma equivalente & 1 & 0 \\
7. Suturou as bordas sem evertê-las & 1 & 0 \\
8. A tensão da sutura foi adequada & 1 & 0 \\
9. O vazamento foi mínimo na anastomose & 1 \\
10. O tempo menor ou igual a 40 minutos & 0 \\
\hline
\end{tabular}


além do refinamento de práticas para diminuir dor e estresse aos animais ${ }^{(13)}$.

Seguindo o princípio de substituição dos animais em pesquisa (replace), o objetivo atual da simulação e treino em cirurgia é desenvolver métodos mais simples e funcionais, utilizando o mínimo possível de animais em experimentação ${ }^{(4)}$, além de usufruir de todo recurso possível que estas técnicas possam oferecer.

Por estas demandas, os modelos de bancada são os mais utilizados pois permitem treinar técnicas cirúrgicas utilizando materiais baratos de forma que a repetição se torna facilitada ao aluno. Estes modelos não apresentam grande realismo, ou seja, em sua maioria, os modelos de bancada são construídos com materiais artificiais tais como borracha, isopor, plástico entre outros, exclusivamente para que o aluno consiga realizar os movimentos da técnica e compreenda a mecânica de seu aprendizado, portanto, não é requerido que o modelo de bancada utilize materiais caros que simulam grandes detalhes da anatomia ou fisiologia relacionada ao procedimento ensinado ${ }^{(3)}$.

Grober et al (2004) ${ }^{(5)}$ questionaram o impacto da fidelidade nos modelos de bancada para o aprendizado de técnicas e seus resultados comprovaram que a aquisição de habilidade cirúrgica em modelos de bancada de baixa fidelidade é tão eficaz quanto em modelos de alta fidelidade como animais vivos.

Para constatação destes resultados, em modelos de simulação os alunos devem ser avaliados por meio de variáveis quantificáveis, como parâmetros que poderão ser medidos conforme o desempenho dos alunos, por exemplo: manuseio do material, aspecto final, falhas no decorrer da técnica, duração do procedimento. Esse tipo de avaliação é fundamental para diferenciar o nível de habilidade de cada indivíduo durante a trajetória do treinamento, comparar suas evoluções na realização da técnica ${ }^{(8)}$. Neste estudo, os parâmetros requisitados aos alunos foram elaborados para contemplar os princípios básicos de uma anastomose patente.

O presente estudo sugeriu a ampliação do uso do modelo de bancada com línguas bovinas, um dos modelos mais usados para ensino de técnicas fundamentais em cirurgia. A inovação envolveu ensinar um procedimento mais avançado e que regularmente não é mostrado aos graduandos em medicina, sendo ensinado principalmente em nível de residência de cirurgia geral e cirurgia vascular.

O procedimento escolhido foi a anastomose arteriovenosa laterolateral pois em nível de residência, diferentes modelos validados, comprovam melhora técnica e cognitiva de residentes após simulações controladas em laboratório(11), estes dados indicam que esta técnica tanto é factível em laboratório quanto requer treinamento devido a sua complexidade.
O modelo utilizado é de baixo custo, fácil preparo e reprodutibilidade. Também permite revisar conceitos básicos de técnica cirúrgica e permite que o aluno aprenda conceitos avançados. Além disso, pode ser usado na graduação e residência para treinamento e aprimoramento de técnicas em cirurgia geral e vascular. A continuação deste estudo com variações do modelo utilizado, ou mudanças no período de treinamento, pode trazer diferentes resultados.

\section{Conclusões}

Comparando o desempenho dos alunos de quinto e décimo semestre, após treinamento específico da anastomose vascular proposta, houve melhora na nota dos dois grupos, mas não houve significância estatística quando os valores foram comparados.

\section{Referências}

1. Reznick RK, MacRae H. Teaching surgical skills—changes in the wind. New Eng J Med. 2006; 355(25):2664-9.

2. Roberts KE, Bell RL, Duffy AJ. Evolution of surgical skills training. World J Gastroenterol. 2006; 12(20):3219-24.

3. Palter V, Grantcharov T. Simulation in surgical education. CMAJ. 2010; 182(11):1191-6.

4. Kinshoku MR, Rodriguez CAL, Fidalgo RS, Duran CCG, Leme PLS, Duarte IS. Uso racional de modelos animais para pesquisa e ensino de microcirurgia. Rev Col Bras Cir. 2012;39(5):414-7.

5. Grober E, Hamstra S, Wanzel K, Reznick R, Matsumoto E, Sidhu R, et al. The educational impact of bench model fidelity on the acquisition of technical skill: the use of clinically relevant outcome measures. Ann Surg. 2004;240(2):374-81.

6. Purim KSM, Santos LDS, Murara GT, Maluf EMCP, Fernandes JW, Skinovsky J. Avaliação de treinamento cirúrgico na graduação de medicina. Rev Col Bras Cir. 2013;40(2):152-6.

7. Franco D, Medeiros J, Grossi A, Franco T. Uso de língua bovina na prática de técnicas de sutura. Rev Col Bras Cir. 2008;35(6):442-4.

8. Okhah Z, Morrissey P, Harrington DT, Cioffi WG, Charpentier KP. Assessment of surgical residents in a vascular anastomosis laboratory. J Surg Res. 2013;185(1), 450-4.

9. Jensen AR, Milner R, Achildi O, Gaughan J, Wilhite DB, Grewal $\mathrm{H}$. Effective instruction of vascular anastomosis in the surgical skills laboratory. Am J Surg. 2008; 19(2)5:189-94.

10. Price J, Naik V, Boodhwani M, Brandys T, Hendry P, Lam BK. A randomized evaluation of simulation training on performance of vascular anastomosis on a high-fidelity in vivo model: the role of deliberate practice. J Thorac Cardiovasc Surg. 2011;142(3):496503.

11. Fann JI, Caffarelli AD, Georgette G, Howard SK, Gaba DM, Youngblood $\mathrm{P}$, et al. Improvement in coronary anastomosis with cardiac surgery simulation. J Thorac Cardiovasc Surg. 2008; 136(6):1486-91.

12. Guimarães MV, Freire JEC, Menezes, LMB. Utilização de animais em pesquisas: breve revisão da legislação no Brasil. Rev Bioet (Impr). 2016;24(2):217-24

13. Raymundo MM, Goldim JR. Ética da pesquisa em modelos animais. Bioética. 2009;10(1):31-44.

Trabalho recebido: $25 / 10 / 2017$

Trabalho aprovado:06/12/2017 